\title{
Facade hollow brick (cobogó) 3D scanning: natural light admission analysis and comparison with original digital 3D model.
}

\section{SIGRADI2018 TECHNOPOLITICAS \\ xxii congresso da sociedade iberoamericana de gráfica digital 22th conference of the iberoamerican society of digital graphics 07|08|09|novembro|2018 iau usp | são carlos | sp br}

\author{
Cristian Vinicius Machado Fagundes \\ PGDesign - UFRGS | Brazil | fagundes.cristian@gmail.com \\ Cauê Duarte Costa \\ PGDesign - UFRGS | Brazil | cauedc@gmail.com \\ Fábio Pinto da Silva \\ DEG/PGDesign/LdSM - UFRGS | Brazil | fabio.silva@ufrgs.br \\ Underléa Miotto Bruscato \\ DA/PGDesign - UFRGS | Brazil | underlea.bruscato@ufrgs.br
}

\begin{abstract}
The cobogó is a hollow brick used for light and ventilation control, besides having an important aesthetic function. With computer graphics, 3d digital models can be used during the design process to verify these functions. Thus, the goal of this paper is to compare and analyze the different digital 3D models obtained (built virtually or through 3D scanning) of a cobogó existing in the Brazilian market, so that visual and lighting differences can be observed, and how these differences can impact the design process.
\end{abstract}

Keywords: Cobogós; 3D Scanning; Parametric Design; Climatic Analysis; 3D model.

\section{INTRODUÇÃO}

\section{совоGó}

Desde que o ser humano se apropriou das técnicas construtivas para construir abrigos para si, ele tentou aproveitar as condições climáticas naturais, mesmo não tendo tecnologias que pudessem substituir condições como luz e vento (Paulert, 2012). Desta forma, controlar essas condições se tornou primordial no comportamento ambiental das construções, como em situações de "admissão seletiva da radiação solar" (Cartana \& Pereira, 2016). Uma forma de controlar a admissão solar é através dos elementos vazados, sendo que um dos mais populares entre eles é o cobogó.

O cobogó foi criado em 1929 por Amadeu Oliveira Coimbra, Ernest August Boeckmann e Antônio de Goés, na cidade de Recife (Vieira et al, 2012). Utilizando as iniciais dos sobrenomes dos autores, o cobogó pode ser definido como "um simples elemento pré-fabricado, próprio a ser construído em série, baseado na vazadura de uma retícula modular sobre uma placa prismática de concreto" (Vieira et al, 2012). Sua propriedade principal é servir como um protetor solar (Paulert, 2012), porém permitindo a passagem de ventilação natural, de forma que é ideal para locais de clima quente com umidade (Vieira et al, 2012).

Com essas propriedades, o cobogó foi utilizado em grande escala na arquitetura brasileira modernista durante o século XX (Pinto et al, 2015). Além das propriedades térmicas e luminotécnicas, o cobogó possui características estéticas para a composição arquitetônica (Vieira et al, 2012), de forma que vem retornando aos projetos de arquitetura e design contemporâneos, utilizando diferentes materiais como cerâmica e vidro, e tendo aplicação em paredes internas e de decoração (Santos, 2016).

Neste contexto, a utilização de iluminação e conforto térmico naturais traz algumas considerações. Pinto et al (2015) entendem que, mesmo com a utilização de cobogós, as edificações se utilizam de sistemas de condicionamento de ar para conforto térmico. Já Barandier (2013) identifica que é necessário ter ganhos de condicionamentos térmicos passivos, ou seja, adequação de projeto aos climas locais, para assim criar uma relação favorável de conforto ambiental através de meios naturais. Mascaró (1991) assinala, ao falar da iluminação natural, que ela não deve gerar desconforto, de forma que os efeitos possam ser controlados através de elementos fixos ou móveis.

\section{TECNOLOGIAS DIGITAIS DE MODELAMENTO E DIGITALIZAÇÃO}

Utilizado em diversas áreas como arquitetura, engenharia, medicina, dentre outros, os sistemas de modelagem tridimensional CAD (computer aided design) são essenciais também para o desenvolvimento de produtos (Foggiatto et al, 2017). Projetados diretamente em softwares de modelagem tridimensional, os modelos podem ser utilizados em simulações, cálculos, desenhos 
de fabricação, prototipagem rápida, dentre outros (Foggiatto et al, 2017).

Outra forma de obtenção desses modelos tridimensionais é utilizando como base a engenharia reversa através da digitalização 3D. A digitalização 3D permite, com o auxílio de softwares e equipamentos próprios, "a captura de curvas, texturas e detalhes de superfícies com grande precisão" (Brendler et al, 2016). Em geral, é feita uma varredura da superfície do objeto para capturar a posição dos pontos e textura, criando, virtualmente, uma nuvem de pontos correspondente ao objeto real (Cardoso et al, 2013). Cartana e Pereira (2016) indicam que essas tecnologias digitais permitem tomadas de decisões tanto na ordem da representação estética do objeto como também integração com simulações computacionais. Com essas possibilidades, diversas empresas que desenvolvem produtos oferecem seus modelos tridimensionais digitais para arquitetos, designers e engenheiros desenvolverem seus projetos (Deca, 2018; Saccaro, 2018).

Entendendo que as características do cobogó permitem ganhos estéticos e luminotécnicos, e que modelos tridimensionais digitais permitem simulações computacionais, o objetivo deste trabalho é averiguar se estes modelos obtidos através da digitalização 3D e da modelagem tradicional de um exemplar específico de cobogó do mercado brasileiro possuem diferenças visuais e luminotécnicas. $\mathrm{O}$ primeiro modelo foi obtido através de da digitalização 3D assistido pela máquina Digimil 3D; já o segundo obteve-se através de um download do modelo tridimensional digital da peça, hospedada em repositório virtual direcionado diretamente do site da fabricante (3D Warehouse, 2018). A partir do momento que os modelos tridimensionais estão cada vez mais sendo utilizados em simulações, se justifica esta pesquisa ao observar que a amostra escolhida já traz uma considerável diferença visual, possibilitando a hipótese de existirem as diferenças supracitadas entre os modelos.

\section{METODOLOGIA}

Pretendendo atingir os objetivos propostos, a metodologia foi organizada da seguinte forma: (1) obtenção e tratamento dos modelos tridimensionais digitais, (2) criação de algoritmo para modelagem de fechamentos externos residenciais, (3) simulações referentes à admissão de iluminação natural e (4) comparações mensuráveis e estético-visuais entre modelos.

\section{OBTENÇÃO DOS MODELOS TRIDIMENSIONAIS DIGITAIS}

Primeiramente, foi escolhido o exemplar do cobogó tanto por conveniência e como por, ao analisar o modelo tridimensional disponibilizado pela empresa, se identificar diferenças visuais consideráveis com a amostra real (Figura 1). Este modelo fornecido (chamado neste artigo de MF) foi acessado em maio de 2017 a partir do site da fabricante, que redirecionava para um repositório virtual da 3D Warehouse. O formato de download foi o .skp, próprio para o programa SketchUp, porém com possibilidade de ser exportado facilmente para outros formatos mais abrangentes como obj. Este modelo também possui características que remetem a uma modelagem tradicional feita diretamente no computador, como planos retos sem rugosidades e wireframe compatível com este tipo de modelagem tridimensional.
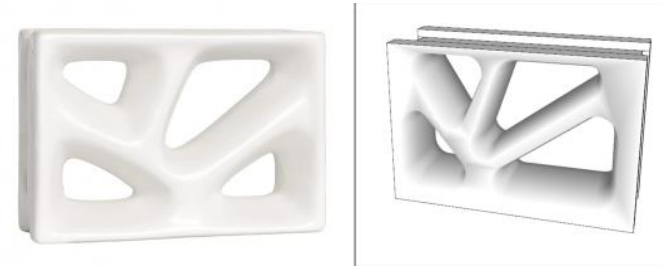

Figura 1: (1) Cobogó real e (2) modelo tridimensional digital fornecido pela empresa; Fonte: http://www.ceramicamartins.com.br/ http://3dwarehouse.sketchup.com/

A seguir, foi feita a aquisição do cobogó para posterior digitalização 3D. O escaneamento da peça de cerâmica vazada (Figura 2) foi através da assistência do equipamento Digimil 3D (instalado no LdSM/UFRGS), que utiliza a tecnologia de digitalização a laser por holografia conoscópica. Este equipamento é um CNC híbrido, com potência máxima de $1 \mathrm{~mW}$, com possíveis trocas de precisão conforme a lente escolhida (Silva, 2011). Como etapa inicial, para eliminar a refletividade do material, utilizou-se na peça cerâmica um spray revelador não aquoso, assim deixando fosca a superfície do material. No estudo foi utilizada a lente de $150 \mathrm{~mm}$, que possui precisão de $0,035 \mathrm{~mm}$. A peça foi digitalizada em cerca de 40min, utilizando a resolução de 0,2 mm. Logo após, as nuvens de pontos geradas foram tratadas no software Geomagic Studio. Ao fim do processo, foi criado o modelo digitalizado, chamado neste artigo de MD.

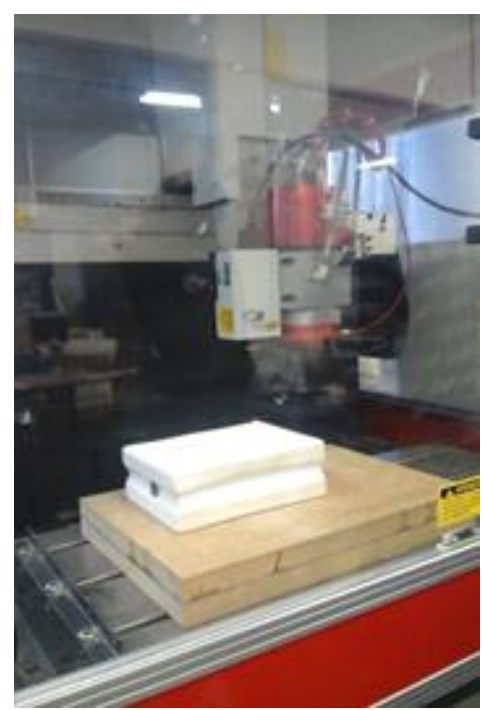

Figura 2: Processo de digitalização 3D. Fonte: Autores.

\section{MODELAGEM DE FECHAMENTOS EXTERNOS}

Nesta etapa, foram modelados através do software Rhinoceros 3D juntamente com o plugin Grasshopper dois fechamentos parametricamente projetados para proteger a entrada de iluminação natural de uma fachada residencial utilizada como exemplo de exercício. Como já descrito, foram utilizados os dois modelos tridimensionais digitais adquiridos de formas distintas. Ambos foram inseridos ao software Rhinoceros 3D. Logo após, criou-se um algoritmo (figura 3) no Grasshopper para replicação parametrizada das peças. 


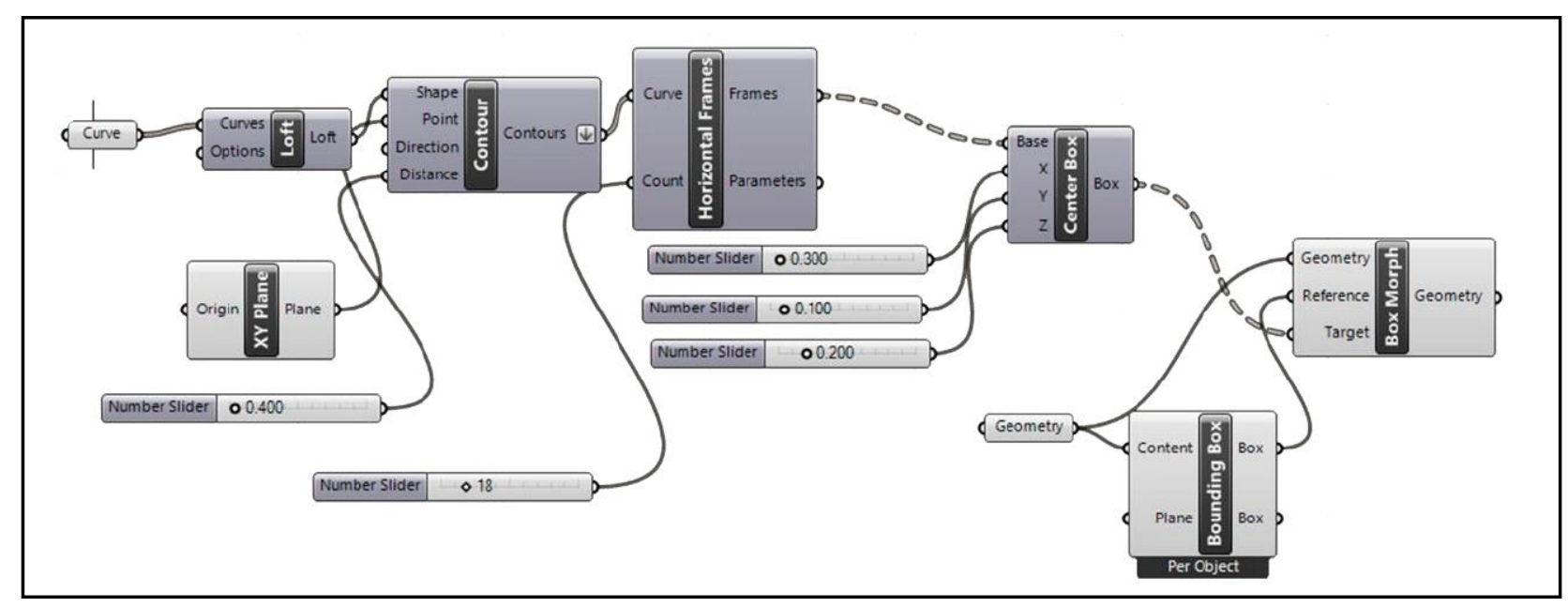

Figura 3: Algoritmo utilizado no controle de replicação da peça. Fonte: Autores.

O algoritmo serviu como base para ambos os fechamentos, sendo organizado pela seguinte forma: desenho das curvas bases no Rhinoceros 3D, criação de uma superfície (loft) através das curvas geradas no Grasshopper, subdivisão horizontais e verticais da superfície (countour e horizontal frames) e por fim inserção das dos modelos na superfície (bounding box e box morph).

\section{SIMULAÇÕES REFERENTES À ADMISSÃO DE ILUMINAÇÃO NATURAL}

Para a realização desta etapa, foram utilizadas imagens geradas através da simulação da incidência solar realizadas pelo plugin DIVA. As mesmas possuem valores em lux (intensidade de iluminação), subdivididas em cores demarcadas pelo grau de intensidade. Deste modo foi possível analisar o grau de iluminância que transpunha ao interior da residência, possibilitando possíveis comparações entre os dois modelos tridimensionais. O estudo foi realizado para a cidade de Porto Alegre - RS (latitude 30 Sul) por motivos de conveniência, sendo que o arquivo climático utilizado no plugin DIVA encontra-se em formato .epw em: <https://energyplus.net/weather>. Os valores dos parâmetros utilizados na simulação para paredes e pisos foram de $30 \%$ de refletância e a condição do ambiente foi demarcada como "clear sky with sun", no dia primeiro de janeiro às 9:00 da manhã, considerando que são medidas pré-definidas pelo plugin.

\section{COMPARAÇÕES MENSURÁVEIS E ESTÉTICO- VISUAIS ENTRE MODELOS.}

Para as comparações mensuráveis, os modelos foram processados no software 3ds Max, e depois levados ao software Geomagic Control X para averiguação das diferenças de medidas. Já as comparações estéticovisuais foram obtidas através da renderização dos modelos no software 3ds Max e plugin V-Ray e posterior análise.

\section{RESULTADOS E SIMULAÇÕES}

Neste item são apresentados e discutidos os resultados das etapas apresentadas na metodologia: (1) digitalização 3D, (2) modelagem de fechamentos externos, (3) simulações referentes à admissão de iluminação natural e (4) comparações mensuráveis e estético-visuais entre modelos.

\section{DIGITALIZAÇÃO 3D}

A digitalização 3D resultou em um modelo de nuvens de pontos em formato .txt, com 947.143 pontos e um tamanho de $23.490 \mathrm{~kb}$. Uma vez que o cobogó praticamente possui um eixo de simetria longitudinal, foi escaneado apenas uma das faces principais. $\mathrm{O}$ arquivo foi carregado no software Geomagic, onde se realizou limpeza, otimização e triangulação da malha. A partir de então, o arquivo passou a ter 9.999 triângulos em um formato .wrp com tamanho de 743 kb (Figura 4).

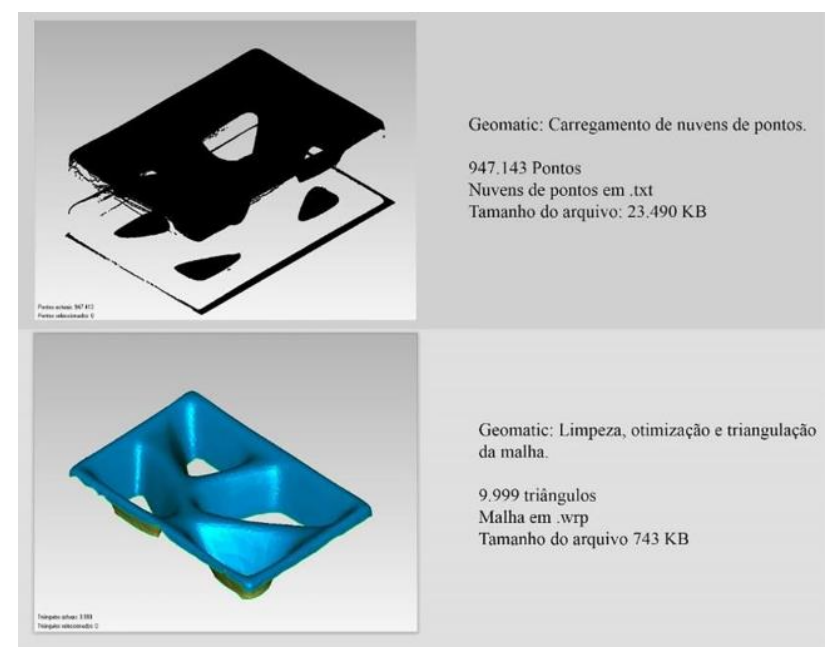

Figura 4: Modelo digitalizado. Fonte: Autores .

O modelo foi então levado ao software 3ds Max, onde foi espelhado (uma vez que só uma das faces foi escaneada) e teve mais algumas correções. No fim do processo, foi exportado um arquivo .obj com tamanho de $1.254 \mathrm{~kb}$. Já o modelo fornecido pela empresa (originalmente em formato .skp) foi exportado diretamente para em .obj, com tamanho final de $105 \mathrm{~kb}$. A diferença de tamanho se explica pela quantidade de vértices e faces do modelo: analisando pelo 3ds Max, enquanto o digitalizado possui 10268 vértices e 20172 faces, o disponibilizado possui 487 vértices e 986 faces. 


\section{MODELAGEM DOS FECHAMENTOS EXTERNOS}

Após a criação do algoritmo, obteve-se no Rhinoceros + Grasshopper um ambiente virtual com um total de 200 elementos cerâmicos vazados, tanto para o modelo digitalizado (MD - Figura 5a) quanto para o modelo fornecido (MF - Figura 5b).

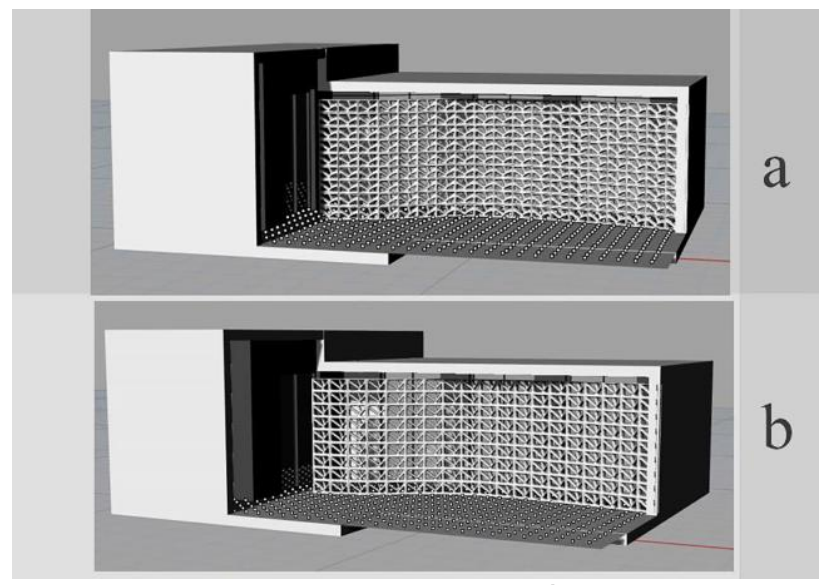

Figura 5: Resultado da modelagem paramétrica. Fonte: Autores.

\section{SIMULACÕES REFERENTES À ADMISSÃO DE ILUMINAÇÃO NATURAL}

Os resultados em lux (índice de iluminância) obtidos nas simulações dos Modelos A (MD) e B (MF) (Figura 6) ressaltam a diferença entre suas distintas formas de aquisição. No Modelo A, a entrada de iluminação se deu em maior amplitude, resultando em diferentes pontos de admissão solar. Mesmo assim, ambos os modelos obtiveram resultados entre 300 a 3000 lux.

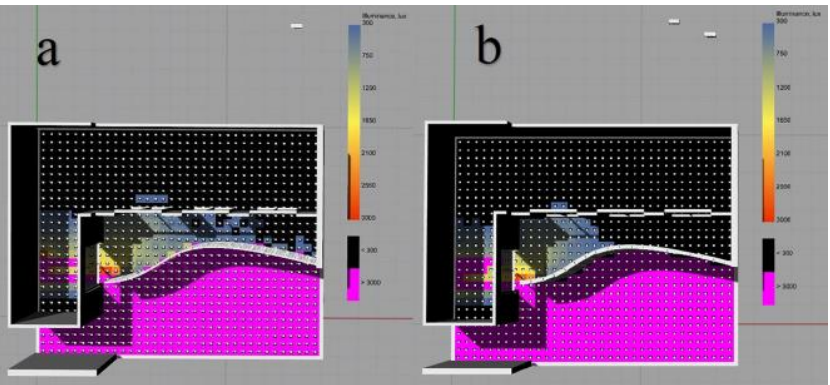

Figura 6: Simulações de admissão da iluminação natural. Fonte: Autores.

Estas diferenças são notadas pela grande diferença entre cheios e vazios de cada peça, e não pelo tamanho propriamente dito de cada uma. Apesar dos vazios aparentemente menores, o MD (a) permitiu, para esta simulação luminotécnica e paramétrica, uma entrada de luminosidade maior que o MF (b). Desta forma, apesar dos resultados semelhantes, já se identifica diferenças numéricas entre os dois modelos digitais.

\section{COMPARAÇÕES MENSURÁVEIS E ESTÉTICO- FORMAIS}

Para a comparação mensurável, foram determinados alguns parâmetros. Entendendo que a principal diferença nos cálculos luminotécnicos se deu pelos cheios e vazios das peças, estes foram o alvo principal das diferenças de medidas, e não dimensão total, por exemplo. Com isso, o MF teve pequenas alterações para que as medidas de ambas as peças fossem similares, uma vez que nem estas dimensões estavam com valores próximos. Para esta adequação, o MF teve reduzido seus valores para $97,8 \%$ no eixo $X, 99,3 \%$ no eixo $Y$ e $93,6 \%$ no eixo $Z$. Além disso, como as peças são simétricas longitudinalmente, foi averiguada a diferença em metade de cada modelo tridimensional digital. Finalmente, foi determinado que o alinhamento se desse pelo centro de cada peça no plano eixo $X Y$ e no ponto mais externo de cada peça no eixo Z. Esta etapa foi desenvolvida toda no software 3ds Max para posterior exportação dos modelos no formato .obj.

A seguir, os modelos foram importados para o software Geomagic Control X, que permite fazer comparações dimensionais. Foi escolhido o MF como referência, enquanto o MD foi sobreposto a este (Figura 7).

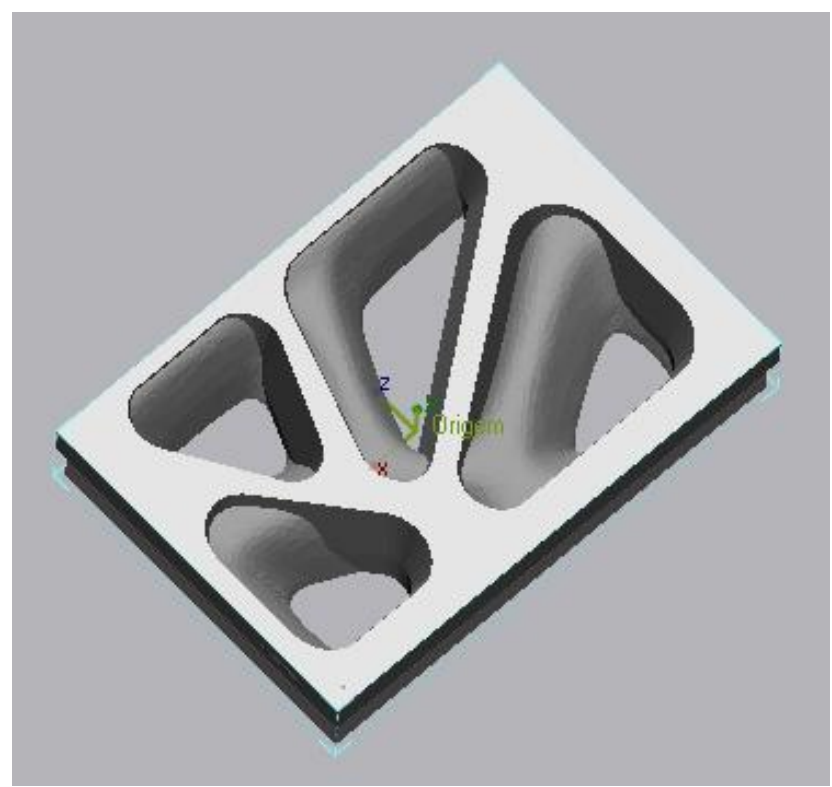

Figura 7: Sobreposição de modelos no software Geomatic Control X. Fonte: Autores.

Uma das ferramentas comparativas utilizadas normalmente é a chamada Comparação $3 D$, que confronta os modelos e identifica os desvios mensuráveis. Porém, os modelos do experimento possuem, visualmente, diferenças consideráveis. Ao utilizar esta ferramenta, os valores encontrados não explicitam com tanta clareza estas diferenças. Portanto, foi escolhida a ferramenta Comparação 2D, que faz a comparação em planos de corte definidos pelos usuários utilizando um mapa de desvio de cores, que é uma forma de visualizar os desvios entre dois modelos digitais (3D Systems, 2017).

Para este experimento, foram definidos cortes longitudinais de 5 em $5 \mathrm{~mm}$ que mostrassem os desvios mensuráveis dos cheios e vazios das peças. As linhas na cor preta são do modelo usado como referência, no caso o MF. Os gráficos gerados em cada corte identificam os desvios: nas cores amarelo a vermelho, os valores são positivos, ou seja, vão em direção ao vazio da peça; os tons azuis identificam valores negativos, ou seja, vão em direção à parte cheia da peça.

Foram identificadas diferenças muito significativas nas peças em todos os cortes longitudinais, tanto para dentro das peças como em direção aos vazios. Já no primeiro corte, a $1 \mathrm{~mm}$ da base (Figura 8), verifica-se 
principalmente que as diferenças são em direção aos vazios (cores amarelo a vermelho), com menos desvios em direção a parte cheia da peça. Além disso, numericamente se identifica diferenças de até $30 \mathrm{~mm}$, o que explicita as diferenças entre as peças. É interessante observar também que os desvios permitem identificar também os cheios e vazios do MD.

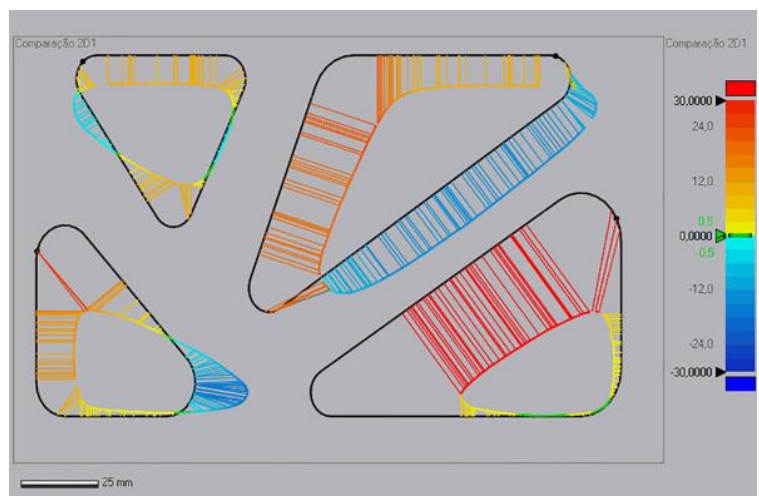

Figura 8: Corte longitudinal a $1 \mathrm{~mm}$ da base. Fonte: Autores.

A partir do corte a $20 \mathrm{~mm}$ (Figura 9), pode se notar que o MF começa a sofrer aumento no tamanho das aberturas. Formalmente é desta forma que o objeto real cobogó se comporta, porém esse aumento não tem correlação com os tamanhos do MD, como pode ser demonstrado na figura 9: há ainda mais diferenças de medidas que no corte a $1 \mathrm{~mm}$, por exemplo.

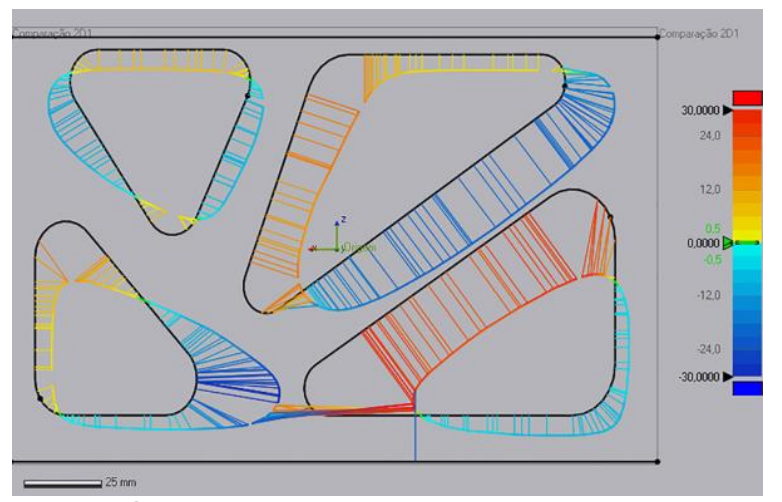

Figura 9: Corte longitudinal a $20 \mathrm{~mm}$ da base. Fonte: Autores.

Continuando os cortes, no plano de $30 \mathrm{~mm}$ (próximo à face mais externa da peça) há continuidade do aumento das aberturas do MF e uma leve similaridade entre as aberturas à direita da imagem (Figura 10). Porém, esta semelhança não condiz com a peça real: tanto a localização como as diferenças de medidas continuam existindo.

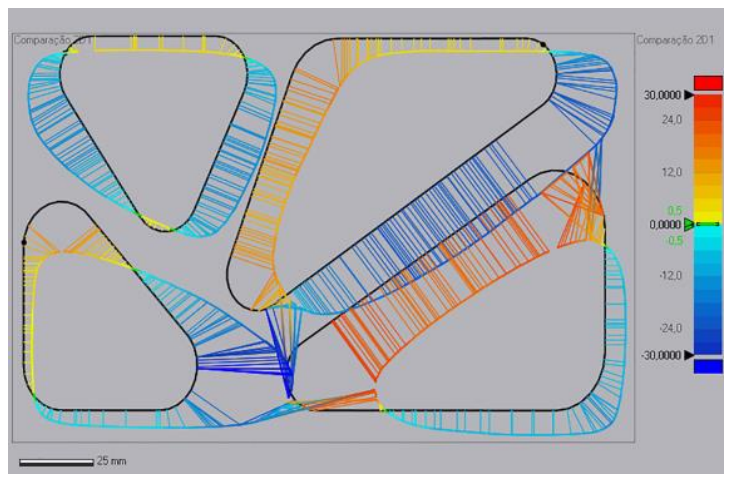

Figura 10: Corte longitudinal a $30 \mathrm{~mm}$ da base. Fonte: Autores.

Finalmente, é interessante destacar o corte a $35 \mathrm{~mm}$ da base (Figura 11). Apesar do MF ainda possuir os vazios marcados, no MD as aberturas chegam ao fim, e se encaminham já para a lateral da peça, identificando uma suavidade maior que no MF.

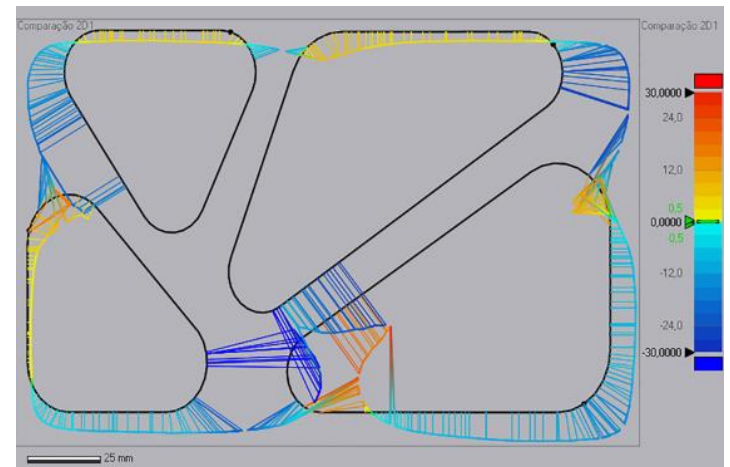

Figura 11: Corte longitudinal a $35 \mathrm{~mm}$ da base. Fonte: Autores.

Além da análise mensural, os objetos foram testados em um ambiente de renderização, para analisar empiricamente as diferenças visuais (Figura 12). Para a renderização, foi utilizado o software 3ds Max junto ao renderizador V-Ray.
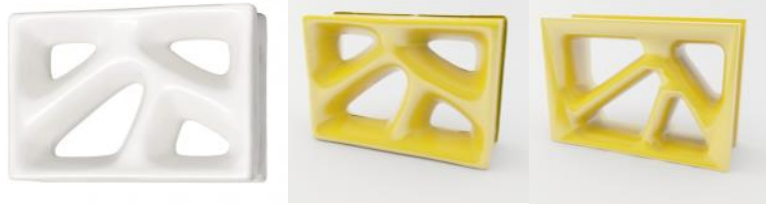

Figura 12: Cobogó real, MD e MF. Fonte: Autores.

Pode se perceber, visualmente, uma grande diferença estética entre os modelos tridimensionais digitais. O MD, à esquerda, mostra-se fiel às linhas e contornos originais da peça, criando um modelo digital realista às características do modelo. Essa é uma das características que a digitalização 3D permite: uma captação tridimensional de peças já existente bastante fidedigna.

Já o MF, fornecido pela empresa, não possui as mesmas semelhanças com o objeto real, perdendo principalmente a questão da organicidade das curvas do cobogó real.

\section{CONCLUSÃO}

Como resultados finais, pode ser observado que 0 modelo digitalizado (MD) possui uma fidelidade de medidas, de estética e de visual em relação ao cobogó real, diferente do modelo fornecido (MF), que possui valores e formas diferentes da real. Com isso, foram encontrados valores distintos nas simulações de iluminação natural e índices de iluminância. As mesmas possuem total importância na etapa de projeto para um resultado fidedigno das necessidades de admissão solar. Entendendo que as tecnologias digitais são importantes nas decisões de projeto (Cartana e Pereira, 2016), se conclui que os modelos digitais utilizados para este fim devem ter diferenças mínimas em relação às peças reais, o que foi verificado apenas no MD e não no MF. 
Além disso, neste caso em particular, o MF apresentou diferenças visuais em relação ao cobogó original, de forma que sua utilização em visualização também é limitada. Com os programas e softwares cada vez mais buscando o fotorrealismo e realidade na representação gráfica, ressalta-se a importância da digitalização 3D para obtenção de modelos tridimensionais digitais com resultados mais fiéis aos cobogós reais.

\section{AGRADECIMENTOS}

Agradecemos ao LdSM/UFRGS pela disponibilidade e apoio durante o processo de digitalização 3D.

\section{REFERÊNCIAS}

3D Systems (2017), Geomagic Control X Tutorial 2D Compare. Acessado https://www.youtube.com/watch?v=TjwRRT5GUqk

3D Warehouse. (2018). Cerâmica Martins. Acessado em https://3dwarehouse.sketchup.com/by/ceramicamartins?nav= models

Barandier, H. (2013). Planejamento e controle ambiental-urbano e a eficiência energética. Rio de Janeiro, PROCEL.

Brendler, C. F., Müller, M. S., Silva, F. P., \& Teixeira F. G. (2016). Uso da digitalização 3D do corpo humano para desenvolvimento de produtos personalizados: Análise comparativa entre os scanners Artec EVA e o Kinect. Estudos em Design | Revista (online), Rio de Janeiro, v. 2, n. $24,24-43$.

Cardoso, E., Santos, S. L., Silva F. P., Teixeira, F. G., \& Silva, T. L. K. (2013). Tecnologias Tridimensionais para Acessibilidade em Museus. Sigradi 2013 - XVII Congreso de la Sociedad Iberoamericana de Gráfica Digital: Diseño basado en Conocimiento, 444-448.
Cartana, R. P. \& Pereira, F. O. R. (2016). Elementos De Controle Solar Desenvolvidos Com Modelagem Paramétrica $E$ Algoritmos Evolutivos. PARC Pesquisa em Arquitetura e Construção, Campinas, SP, v. 7, n. 3, 133-144

Deca. (2018). Arquivos 2D e 3D para Projetos. Acessado em https://www.deca.com.br/biblioteca/arquivos-2d-e-3d-paraprojetos/

Foggiatto, J. A., Volpato, N. \& Bontorin, A. C. B. (2007). Recomendações Para Modelagem Em Sistemas Cad-3d. Anais 40 Congresso Brasileiro de Engenharia de Fabricação.

Mascaró, L.. (1991). Energia Na Edificação: Estratégias Para Minimizar Seu Consumo. São Paulo.

Paulert, R. (2012). Uso De Elementos Vazados Na Arquitetura: Estudo De Três Obras Educacionais Contemporâneas. Dissertação de Mestrado, Curitiba.

Pinto, H. R. S., Queiroz, N., Cardoso, A. R. B. \& Sousa, J. P. M. (2015) XIX Congresso da Sociedade Ibero-americana de Gráfica Digital 2015, vol. 2 n.3, Florianópolis.

Saccaro (2018). Catálogos 3d. Acessado em http://www.saccaro.com.br/pt-br/catalogos/catalogo-3d

Santos, D. F. S. (2016). Arquitetura bioclimática: a integração do cobogó ao ambiente construído como ferramenta geradora de conforto térmico e lumínico em regiões quentes e úmidas. 5 SICS - Seminário Internacional de Construções Sustentáveis, Passo Fundo / RS. Anais V SICS.

Silva, F. P. (2011). Usinagem de espumas de poliuretano e digitalização tridimensional para fabricação de assentos personalizados para pessoas com deficiência. 2011. 192 f. Tese (Doutorado) - Curso de Pós-graduação em Engenharia Minas, Metalúrgica e de Materiais, Universidade Federal do Rio Grande do Sul, Porto Alegre.

Vieira A., Borba, C. \& Rodrigues J. (2012). Cobogó De Pernambuco. Recife 